\title{
Effect of Sorghum Water Extract Type, Concentration and Application Time on Weed Density and Yield of Wheat
}

\author{
*Muhammad Hamid, Shad khan Khalil, Nawab Ali, Muhammad Mehran Anjum and Muhammad Aatif \\ Department of Agronomy Faculty of Crop Production Sciences, University of Agriculture, Pakistan
}

Submission: February 07, 2017; Published: March 07, 2017

*Corresponding author: Muhammad Hamid, Department of Agronomy Faculty of Crop Production Sciences, University of Agriculture, Peshawar, Pakistan

\begin{abstract}
A field experiment entitled "Effect of sorghum water extract type, concentration and application time on weed density and yield of wheat" was conducted at agronomy research Farm, The University of Agriculture Peshawar, Pakistan during winter season 2014-15. The experiment was laid out in a randomized complete block design (RCBD) having three replication. A plot size of $3 \mathrm{~m} \times 1.8 \mathrm{~m}$ (L x W) was used. Each plot was consist of six rows having $30 \mathrm{~cm}$ row to row distance. Different concentration (1:3) i.e. (1 kg plant herbage and 3 liters of water), (1:4) i.e. (1 kg plant herbage and 4 liters of water) and 1:5 (1 kg plant herbage and 5 liters of water) of sorghum stem and leaf extract were sprayed at three stages full at Feekes 1 (Emergence), full at Feekes 2 (Tillering), and half at Feekes 1 (Emergence) plus half at Feekes 2 (Tillering). The experiment was also comprised of control (no weed control), hand weeding and herbicides application. All the weed control treatments significantly affected weed density, productive tillers, non productive tillers, thousand grain weight, biological yield and grain yield. Herbicide sprayed plots produced minimum (24) number of weeds, maximum productive tillers m-2 (379), thousand grain weight (51 g) biological yield (10558 kg ha-1) and grain yield (3976 kg ha-1) as compared to control. In case of sorghum extract type leaf water extract produced minimum (131) weeds, maximum (339) productive tillers $\mathrm{m}-2$, thousand grain weight (44.62 g), biological yield (9399 kg ha-1) and grain yield (3797 kg ha-1) as compared to stem water extract. Sorghum water extract at higher concentration (1:3) produced minimum (86) number of weeds, maximum number of productive tillers (350), thousand grain weight (47.17 g), biological yield (9339 kg ha-1) and grain yield (3575 kg ha-1) as compared with lower concentration (1:5).It is concluded that the use of farm grown sorghum for weed control is less costly, farmers friendly and organic method of weed control that can be explored as cheap method for increasing wheat yield in weedy fields.
\end{abstract}

Keywords: Sorghum; Water Extract; Weed Density and Yield of Wheat

\section{Introduction}

Wheat (Triticum aestivum) L. is an annual self pollinated determinate long day crop. It is hardy crop that can grow in a wide range of environmental conditions. More of the earth's surface is covered by wheat than by any other food crop. Its production is third largest cereal production in the world, after maize and rice. In terms of dietary intake, wheat comes second to rice as a main food crop. Currently, about 65 percent of the wheat crop is used for food (FAO, 2013). It is the most important cereal Hussain et al. (2010) and also major staple food of Pakistan Blackshaw [1], Aslam et al. [2]. Wheat adds a big share towards the country's GDP Aslam et al. [2].

Agriculture is the backbone of Pakistan's economy. It contributes about 21 percent of our GDP. Wheat is the essential diet of population among the important crops of Pakistan. It occupies the central position in agriculture policies of the government and contributes $2.2 \%$ to GDP. Area under cultivation of wheat in Pakistan is 8693 thousand ha, production is 24231 thousand tones and the yield is $2787 \mathrm{~kg}$ ha-1 MINFA [3].

In Pakistan, weeds pose serious problems in crop production as weed control is ignored due to lack of education and poor financial resources of the farmers. Small farmers cannot afford the cost of weed management practices for crop production. Thus weeds growing among crop plants adversely affect yield and quality of the harvest and increase production costs, resulting in high economic losses. Some species of weed plants might be a serious threat to crop plants diversity, sharing nutrients, moisture, sun light and space Ozturk et al. [4]. Weeds compete with crop plants for nutrients, solar radiation, water, carbon dioxide and space. Apart from competition, many other factors like lodging and harvesting problem are created by weeds. In Pakistan, weeds are accountable for 30 percent grain yield losses 
in wheat which amounts to Rs.1150 million annually Marwat et al. [5]. Additionally, the risk of weed resistance development and high cost-benefit ratio are other disadvantages of herbicides Kordali et al. [6].

Therefore in recent years, new approaches such as plant allelopathic effects have been considered to suppress weeds in agricultural systems Dayan et al. (2009). Allelopathy is a natural, inexpensive, environmentally safe and an organic approach to control weeds and increase crop yields while conserving the ecosystem. The phenomenon of allelopathy can be practically utilized for weed control in the form of crop rotations, intercropping, allelopathic mulches, and spray of allelopathic plant water extracts Bhowmik and Inderjit [7], Jabran et al. [8], Farooq et al. [9]. Thus many plant based compounds may play a vital role as fertilizer or bio-herbicide for neighboring plants. Allelopathic characteristics of many plant species including weeds, crops and trees need to be investigated for possible use in weed management programs. Thus the newly identified allelochemicals might be helpful to formulate and commercialize new plants derived herbicides. Blackshaw et al. [1] observed that farmers are interested to explore more attractive and inclusive weed control strategies that minimize the serious weed infestation by time and in the use of low rate of synthetic herbicides with lower production costs. Allelopathic plants for example sorghum, sunflower and oats, contain a number of allelochemicals in low quality act as hormones and in high amount as herbicides. Allelopathic crop plants not only control weeds but also enhance crop growth and yield. Keeping in view the losses due to weeds, resistance creating to herbicides application and the recognized importance of allelochemicals in weed management, a present field experiment will be conducted under the agro climatic condition of Peshawar.

\section{Materials and Methods}

A field experiment entitled response of weed density and yield of wheat to sorghum extract type, concentration, and application time was conducted at Agriculture Research Farm, The University of Agriculture Peshawar, Pakistan during winter season 201415 [10]. The experiment was laid out in a randomized complete block design (RCBD) having three replication. A plot size of $3 \mathrm{~m} \mathrm{x}$ $1.8 \mathrm{~m} \mathrm{(L} \mathrm{x} \mathrm{W)} \mathrm{was} \mathrm{used.} \mathrm{Each} \mathrm{plot} \mathrm{was} \mathrm{consist} \mathrm{of} \mathrm{six} \mathrm{rows} \mathrm{having}$ $30 \mathrm{~cm}$ row to row distance. Seed was sown at the rate of 120 $\mathrm{kg} \mathrm{ha}^{-1}$. The nitrogen and phosphorous fertilizer were applied at the rate of $100-90 \mathrm{~kg} \mathrm{ha}^{-1}$ respectively according to the general recommendations of wheat and source of fertilizer was urea $(46 \% \mathrm{~N})$ and diammonium phosphate $\left(46 \% \mathrm{P}^{2} \mathrm{O}_{5}\right.$ and $\left.18 \% \mathrm{~N}\right)$. All phosphorus fertilizer and one-third of nitrogen were applied at the time of sowing and remaining nitrogen was applied in splits [11]. Different concentration (1:3) i.e. (1 kg plant herbage and 3 liters of water), (1:4) i.e. (1 kg plant herbage and 4 liters of water) and 1:5 (1 kg plant herbage and 5 liters of water) of sorghum stem and leaf extract was sprayed at three stages full at Feekes 1 (Emergence), full at Feekes 2 (Tillering), and half at Feekes 1 (Emergence) plus half at Feekes 2 (Tillering). The experiment was also comprised of control (no weed control), hand weeding and herbicides application. All other agronomic practices were applied uniformly.

\section{Preparation of Sorghum Herbage Water Extracts}

Mature plant herbage (stem and leaf) of sorghum was collected from New Developmental Farm, The University of Agriculture Peshawar, Pakistan [12]. This herbage was used in water extracts preparation for field experiment. For making sorghum water extract, the sorghum stems and leaves were separated and sun dried for two days. After sun drying the stem and leave were chopped separately with electric fodder chopping machine. The stem and leaf material were soaked in water separately for 48 hours by maintaining (1:3) i.e. (1 kg plant herbage and 3 liters of water), (1:4) i.e. (1 kg plant herbage and 4 liters of water) and 1:5 (1 kg plant herbage and 5 liters of water) for preparation of different concentrations.

\section{i.Sorghum Extract Type}
a) Leaf Water Extract (LWE)
b) Stem Water Extract (SWE)

\section{ii.Concentration (C)}

a) $\quad \mathrm{C}_{1}=1: 3 \quad(1 \mathrm{~kg}$ sorghum plant herbage and 3 liters of water)

b) $\mathrm{C}_{2}=1: 4 \quad(1 \mathrm{~kg}$ sorghum plant herbage and 4 liters of water)

c) $\mathrm{C}_{3}=1: 5 \quad(1 \mathrm{~kg}$ sorghum plant herbage and 5 liters of water)

\section{iii.Application Time (AT)}
a) $\quad \mathrm{AT}_{1}=$ Feekes 1 (Emergence)
b) $\quad \mathrm{AT}_{2}=$ Feekes 2 (Tillering)
c) $\mathrm{AT}_{3}=$ Half at Feekes 1 (Emergence) plus half at Feekes 2 (Tillering)

\section{Results}

\section{Weeds density $\left(\mathrm{m}^{-2}\right)$}

Statistical analysis of the data showed that sorghum extract type (SET), Concentration(C), application time (AT), control vs. treated, hand weeding (HW) vs. control and herbicide application (HA) vs. treated have significant effect on number of weeds $\mathrm{m}^{-2}$, while all possible interactions except $\mathrm{C} x \mathrm{AT}$ were non-significant (Table 1). Mean values for sorghum water extract (SWE) concentration showed that higher concentration (1:3) resulted in less number of weeds $\left(86 \mathrm{~m}^{-2}\right)$. Decreasing concentration increased number of weeds and maximum number of weeds (162 m-2) at 1:5. HW plot produced significantly less number of weeds $\left(24 \mathrm{~m}^{-2}\right)$ as compared with SWE $\left(134 \mathrm{~m}^{-2}\right)$. Whereas the plot treated with SWE produced significantly less number 
of weeds $\left(134 \mathrm{~m}^{-2}\right)$ as compared with control plot (183 m-2). In case of SET the mean values showed that minimum number of weeds $\left(131 \mathrm{~m}^{-2}\right)$ was produced by the plot treated with leaf water extract (LWE), whereas the maximum number of weeds per $\mathrm{m} 2\left(138 \mathrm{~m}^{-2}\right)$ was produced by plot treated with stem water extract (SWE). Mean values for AT showed that SWE applied at emergence produced minimum number of weeds m-2 (129) while maximum number of weeds $m-2$ 142) was recorded when SWE applied half at emergence and half at tillering.

\section{Productive Tillers $\mathbf{m}^{-2}$}

Table 1: Weed density $\left(\mathrm{m}^{-2}\right)$ of wheat as affected by sorghum water extract type, concentration and application time.

\begin{tabular}{|c|c|c|c|c|}
\hline \multirow{2}{*}{$\begin{array}{c}\text { Concentration } \\
\text { Herbage(kg):Water(L) }\end{array}$} & \multirow{2}{*}{ Application Time } & \multicolumn{2}{|c|}{ Extract Type } & \multirow{2}{*}{ Mean } \\
\hline & & Leaf water extract & Stem water extract & \\
\hline $1: 3$ & Emergence (E) & 75 & 86 & 81 \\
\hline $1: 3$ & Tillering (T) & 67 & 78 & 72 \\
\hline $1: 3$ & $50 \%$ at $\mathrm{E}+50 \%$ at $\mathrm{T}$ & 104 & 105 & 105 \\
\hline $1: 4$ & Emergence (E) & 141 & 158 & 150 \\
\hline $1: 4$ & Tillering (T) & 156 & 160 & 158 \\
\hline $1: 4$ & $50 \%$ at $\mathrm{E}+50 \%$ at $\mathrm{T}$ & 155 & 157 & 156 \\
\hline $1: 5$ & Emergence (E) & 157 & 159 & 158 \\
\hline $1: 5$ & Tillering (T) & 161 & 167 & 164 \\
\hline $1: 5$ & $50 \%$ at $\mathrm{E}+50 \%$ at $\mathrm{T}$ & 162 & 169 & 166 \\
\hline $1: 3$ & & 82 & 90 & 86 \\
\hline $1: 4$ & & 151 & 159 & 155 \\
\hline \multirow[t]{4}{*}{$1: 5$} & & 160 & 165 & 162 \\
\hline & \multirow{3}{*}{$\begin{array}{c}\text { Emergence }(\mathrm{E}) \\
\text { Tillering }(\mathrm{T}) \\
50 \% \text { at } \mathrm{E}+50 \% \text { at } \mathrm{T}\end{array}$} & 124 & 135 & 129 \\
\hline & & 128 & 135 & 131 \\
\hline & & 140 & 144 & 142 \\
\hline \multicolumn{2}{|c|}{ Mean } & 131 & 138 & \\
\hline \multicolumn{2}{|c|}{ Control } & 183 & & \\
\hline \multicolumn{2}{|c|}{ Sorghum water extract } & 134 & & \\
\hline \multicolumn{2}{|c|}{ Hand weeding } & 24 & & \\
\hline \multicolumn{2}{|c|}{ Sorghum water extract } & 134 & & \\
\hline \multicolumn{2}{|c|}{ Herbicide application } & 29 & & \\
\hline & & 134 & & \\
\hline
\end{tabular}

LSD value for concentration at $\mathrm{P} \leq 0.05=1.301921$.

LSD value for Application time at $P \leq 0.05=1.301921$.

Mean sharing the same letters are non significant at $\mathrm{P} \leq 0.05$ using LSD Test.

Statistical analysis of the data revealed that sorghum extract type (SET), Concentration (C) application time (AT), control vs. treated, hand weeding (HW) vs. treated and herbicide application (HA) vs. treated significantly affected number of productive tillers $\mathrm{m}^{-2}$, while all possible interactions were nonsignificant (Table 1). Mean values for sorghum water extract
(SWE) concentration showed that higher concentration (1:3) resulted in more productive tillers $\mathrm{m}^{-2}$ (350). Decreasing concentration decreased productive tillers and minimum tillers $\left(317 \mathrm{~m}^{-2}\right)$ were recorded at 1:5. HW plot produced significantly more number of productive tillers $\mathrm{m}^{-2}(372)$ as compared with SWE $\left(334 \mathrm{~m}^{-2}\right)$. Whereas the plot treated with SWE produced 
significantly more number of productive tillers m-2 (334) as compared with control plot $\left(282 \mathrm{~m}^{-2}\right)$. In case of SET the mean values showed that maximum number of productive tillers $\mathrm{m}^{-2}$ (339) was produced by the plot treated leaf water extract (LWE), while minimum number of productive tillers m-2 (329) was produced by plot treated with stem water extract (SWE) (Tables $1 \& 2)$.

Table 2: Productive tillers $\mathrm{m} 2$ of wheat as affected of sorghum water extract type, concentration and application time.

\begin{tabular}{|c|c|c|c|c|}
\hline \multirow{2}{*}{$\begin{array}{c}\text { Concentration } \\
\text { Herbage(kg):Water(L) }\end{array}$} & \multirow{2}{*}{ Application Time } & \multicolumn{2}{|c|}{ Extract Type } & \multirow{2}{*}{ Mean } \\
\hline & & Leaf water extract & Stem water extract & \\
\hline $1: 3$ & Emergence (E) & 359 & 352 & 355 \\
\hline $1: 3$ & Tillering (T) & 354 & 350 & 352 \\
\hline $1: 3$ & $50 \%$ at $\mathrm{E}+50 \%$ at $\mathrm{T}$ & 346 & 340 & 343 \\
\hline $1: 4$ & Emergence (E) & 342 & 336 & 339 \\
\hline $1: 4$ & Tillering (T) & 341 & 333 & 337 \\
\hline $1: 4$ & $50 \%$ at $\mathrm{E}+50 \%$ at $\mathrm{T}$ & 334 & 322 & 328 \\
\hline $1: 5$ & Emergence (E) & 329 & 314 & 322 \\
\hline $1: 5$ & Tillering (T) & 328 & 313 & 321 \\
\hline $1: 5$ & $50 \%$ at $\mathrm{E}+50 \%$ at $\mathrm{T}$ & 316 & 304 & 310 \\
\hline $1: 3$ & & 353 & 347 & $350 \mathrm{a}$ \\
\hline $1: 4$ & & 339 & 330 & $335 \mathrm{~b}$ \\
\hline \multirow[t]{4}{*}{$1: 5$} & & 324 & 311 & $317 \mathrm{c}$ \\
\hline & \multirow{3}{*}{$\begin{array}{c}\text { Emergence }(\mathrm{E}) \\
\text { Tillering }(\mathrm{T}) \\
50 \% \text { at } \mathrm{E}+50 \% \text { at } \mathrm{T}\end{array}$} & 344 & 334 & $339 a$ \\
\hline & & 341 & 332 & $337 \mathrm{a}$ \\
\hline & & 332 & 322 & $327 \mathrm{~b}$ \\
\hline \multicolumn{2}{|c|}{ Mean } & 339 a & & \\
\hline \multicolumn{2}{|c|}{ Control } & $282 \mathrm{~b}$ & & \\
\hline \multicolumn{2}{|c|}{ Sorghum water extract } & $334 \mathrm{a}$ & & \\
\hline \multicolumn{2}{|c|}{ Hand weeding } & $372 \mathrm{a}$ & & \\
\hline \multicolumn{2}{|c|}{ Sorghum water extract } & $334 \mathrm{~b}$ & & \\
\hline \multirow{2}{*}{\multicolumn{2}{|c|}{ Herbicide application }} & $379 \mathrm{a}$ & & \\
\hline & & $334 \mathrm{~b}$ & & \\
\hline
\end{tabular}

LSD value for concentration at $P \leq 0.05=6.992622$.

LSD value for Application time at $P \leq 0.05=6.992622$.

Mean sharing the same letters are non significant at $P \leq 0.05$ using LSD Test.

Mean values for AT showed that SWE applied at emergence produced maximum number of productive tillers $\mathrm{m}^{-2}\left(339 \mathrm{~m}^{-2}\right)$, while minimum number of productive tillers $\left(327 \mathrm{~m}^{-2}\right)$ was recorded when SWE was applied half at emergence and half at tillering stage.

\section{Non-productive Tillers $\mathbf{m}-2$}

Statistical analysis of the data revealed that sorghum extract type (SET), Concentration(C) application time (AT), control vs. treated, hand weeding (HW) vs. treated and herbicide application (HA) vs. treated significantly affected number of non-productive tillers $\mathrm{m}-2$, while all possible interactions were found non-significant (Table 1). Mean values for sorghum water extract (SWE) concentration showed that higher concentration (1:3) resulted in less number of non productive tillers $m-2$ (25).
Decreasing concentration increased number of non productive tillers $\mathrm{m}^{-2}$ and maximum tillers $\left(32 \mathrm{~m}^{-2}\right)$ were recorded at 1:5. Hand weeding ( $\mathrm{HW}$ ) plot produced significantly less number of non productive tillers m-2 (23) as compared with SWE (29 m-2). Whereas the plot treated with SWE produced significantly less number of non productive tillers m-2 (29) as compared with control plot $\left(34 \mathrm{~m}^{-2}\right)$. In case of SET the mean values showed that less number of non-productive tiller $\mathrm{m}^{-2}$ (28) was produced by the plot treated with leaf water extract (LWE), whereas the maximum number of non-productive tillers $\mathrm{m}^{-2}(30)$ was produced by plot treated with stem water extract (SWE). Mean values for AT showed that SWE applied at emergence produced less number of non-productive tillers $\mathrm{m}^{-2}(27)$, while highest number of non-productive tillers $\mathrm{m}^{-2}$ (31) was recorded when SWE applied half at emergence and half at tillering stage. 


\section{Days to Physiological Maturity}

Table 3: Nonproductive tillers $\mathrm{m} 2$ of wheat as affected of sorghum water extract type, concentration and application time.

\begin{tabular}{|c|c|c|c|c|}
\hline \multirow{2}{*}{$\begin{array}{c}\text { Concentration } \\
\text { Herbage(kg):Water(L) }\end{array}$} & \multirow{2}{*}{ Application Time } & \multicolumn{2}{|c|}{ Extract Type } & \multirow{2}{*}{ Mean } \\
\hline & & Leaf water extract & Stem water extract & \\
\hline $1: 3$ & Emergence (E) & 23 & 24 & 24 \\
\hline $1: 3$ & Tillering (T) & 25 & 26 & 26 \\
\hline $1: 3$ & $50 \%$ at $\mathrm{E}+50 \%$ at $\mathrm{T}$ & 26 & 27 & 26 \\
\hline $1: 4$ & Emergence (E) & 27 & 28 & 28 \\
\hline $1: 4$ & Tillering (T) & 28 & 30 & 29 \\
\hline $1: 4$ & $50 \%$ at $\mathrm{E}+50 \%$ at $\mathrm{T}$ & 29 & 34 & 32 \\
\hline $1: 5$ & Emergence (E) & 28 & 33 & 31 \\
\hline $1: 5$ & Tillering (T) & 32 & 34 & 33 \\
\hline $1: 5$ & $50 \%$ at $\mathrm{E}+50 \%$ at $\mathrm{T}$ & 33 & 37 & 35 \\
\hline $1: 3$ & & 25 & 26 & 25 \\
\hline $1: 4$ & & 28 & 31 & 29 \\
\hline \multirow[t]{6}{*}{$1: 5$} & & 31 & 34 & 33 \\
\hline & \multirow{4}{*}{$\begin{array}{c}\text { Emergence }(\mathrm{E}) \\
\text { Tillering }(\mathrm{T}) \\
50 \% \text { at } \mathrm{E}+50 \% \text { at } \mathrm{T}\end{array}$} & 26 & 28 & 27 \\
\hline & & 28 & 30 & 29 \\
\hline & & 29 & 32 & 31 \\
\hline & & & & \\
\hline & Mean & 28 & & \\
\hline \multicolumn{2}{|c|}{ Control } & 34 & & \\
\hline \multicolumn{2}{|c|}{ Sorghum water extract } & 29 & & \\
\hline & Hand weeding & 23 & & \\
\hline \multirow{2}{*}{\multicolumn{2}{|c|}{$\begin{array}{l}\text { Sorghum water extract } \\
\text { Herbicide application }\end{array}$}} & 29 & & \\
\hline & & 22 & & \\
\hline & Sorghum water extract & 29 & & \\
\hline
\end{tabular}

LSD value for concentration at $P \leq 0.05=2.753949$.

LSD value for Application time at $\mathrm{P} \leq 0.05=2.753949$.

Mean sharing the same letters are non significant at $P \leq 0.05$ using LSD test.

Statistical analysis of the data showed that sorghum extract type (SET), concentration (C), application time (AT), control vs. treated, hand weeding (HW) vs. treated and herbicide application (HA) vs. treated had no significant effect on days to physiological maturity. All the possible interactions were also found non-significant. Herbicide application, hand weeding and control plots resulted same (155 days) in comparison with

Table 4: Days to physiological maturity of wheat as affected by sorghum water extract type, concentration and application time.

\begin{tabular}{|c|c|c|c|c|}
\hline \multirow{2}{*}{$\begin{array}{c}\text { Concentration } \\
\text { Herbage(kg):Water(L) }\end{array}$} & \multirow{2}{*}{ Application Time } & \multicolumn{2}{|c|}{ Extract Type } & \multirow{2}{*}{ Mean } \\
\hline & & Leaf water extract & Stem water extract & \\
\hline $1: 3$ & Emergence (E) & 154 & 155 & 155 \\
\hline $1: 3$ & Tillering (T) & 155 & 155 & 155 \\
\hline $1: 3$ & $50 \%$ at $\mathrm{E}+50 \%$ at $\mathrm{T}$ & 155 & 155 & 155 \\
\hline $1: 4$ & Emergence (E) & 155 & 156 & 156 \\
\hline $1: 4$ & Tillering (T) & 155 & 155 & 155 \\
\hline $1: 4$ & $50 \%$ at $\mathrm{E}+50 \%$ at $\mathrm{T}$ & 156 & 155 & 156 \\
\hline
\end{tabular}

sorghum water extract treated plots (155 days). Mean values for sorghum water extract (SWE) concentration showed that higher and lower concentration resulted same days (155). Mean values for AT showed the same result for application at emergence and tillering stage (155 days). Also sorghum leaf water extract and stem water extract resulted same (155 days) (Tables 3 \& 4). 
International Journal of Environmental Sciences \& Natural Resources

\begin{tabular}{|c|c|c|c|c|}
\hline $1: 5$ & Emergence (E) & 155 & 156 & 156 \\
\hline $1: 5$ & Tillering (T) & 155 & 156 & 156 \\
\hline $1: 5$ & $50 \%$ at $\mathrm{E}+50 \%$ at $\mathrm{T}$ & 155 & 155 & 155 \\
\hline $1: 3$ & & 155 & 155 & 155 \\
\hline $1: 4$ & & 155 & 155 & 155 \\
\hline \multirow[t]{5}{*}{$1: 5$} & & 155 & 155 & 155 \\
\hline & \multirow{4}{*}{$\begin{array}{c}\text { Emergence }(\mathrm{E}) \\
\text { Tillering }(\mathrm{T}) \\
50 \% \text { at } \mathrm{E}+50 \% \text { at } \mathrm{T}\end{array}$} & 155 & 156 & 155 \\
\hline & & 155 & 155 & 155 \\
\hline & & 155 & 155 & 155 \\
\hline & & & & \\
\hline \multicolumn{2}{|c|}{ Mean } & 155 & 155 & \\
\hline \multicolumn{2}{|c|}{ Control } & 156 & & \\
\hline \multicolumn{2}{|c|}{ Sorghum water extract } & 155 & & \\
\hline \multicolumn{2}{|c|}{ Hand weeding } & 155 & & \\
\hline \multicolumn{2}{|c|}{ Sorghum water extract } & 155 & & \\
\hline \multicolumn{2}{|c|}{ Herbicide application } & 155 & & \\
\hline \multicolumn{2}{|c|}{ Sorghum water extract } & 155 & & \\
\hline
\end{tabular}

LSD value for concentration at $P \leq 0.05=N S$.

LSD value for Application time at $\mathrm{P} \leq 0.05=\mathrm{NS}$.

Mean sharing the same letters are non significant at $P \leq 0.05$ using LSD test.

\section{Grain fill duration}

Statistical analysis of the data showed that sorghum extract type (SET), concentration (C), application time (AT), control vs. treated, hand weeding (HW) vs. treated and herbicide application (HA) vs. treated had no significant effect on grain fill duration. All the possible interactions were also found nonsignificant. Herbicide application, hand weeding and control plots resulted same duration (34 days) in comparison with sorghum water extract treated plots (34 days). Mean values for sorghum water extract (SWE) concentration showed that higher and lower concentration resulted same days (34). Mean values for AT showed the same result for application at emergence and tillering stage (34 days). Also sorghum leaf water extract and stem water extract resulted same (34 days).

\section{Thousand grain yield $(\mathrm{g})$}

Table 5: Grain fill duration of wheat as affected by sorghum water extract type, concentration and application time.

\begin{tabular}{|c|c|c|c|c|}
\hline \multirow{2}{*}{$\begin{array}{c}\text { Concentration } \\
\text { Herbage(kg):Water(L) }\end{array}$} & \multirow{2}{*}{ Application Time } & \multicolumn{2}{|c|}{ Extract Type } & \multirow{2}{*}{ Mean } \\
\hline & & Leaf water extract & Stem water extract & \\
\hline $1: 3$ & Emergence (E) & 35 & 34 & 34 \\
\hline $1: 3$ & Tillering $(\mathrm{T})$ & 34 & 34 & 34 \\
\hline $1: 3$ & $50 \%$ at $\mathrm{E}+50 \%$ at $\mathrm{T}$ & 34 & 34 & 34 \\
\hline $1: 4$ & Emergence (E) & 33 & 34 & 34 \\
\hline $1: 4$ & Tillering (T) & 34 & 34 & 34 \\
\hline $1: 4$ & $50 \%$ at $\mathrm{E}+50 \%$ at $\mathrm{T}$ & 34 & 34 & 34 \\
\hline $1: 5$ & Emergence (E) & 34 & 34 & 34 \\
\hline $1: 5$ & Tillering (T) & 34 & 34 & 34 \\
\hline $1: 5$ & $50 \%$ at $\mathrm{E}+50 \%$ at $\mathrm{T}$ & 33 & 33 & 33 \\
\hline $1: 3$ & & 34 & 34 & 34 \\
\hline $1: 4$ & & 34 & 34 & 34 \\
\hline \multirow[t]{5}{*}{$1: 5$} & & 34 & 34 & 34 \\
\hline & \multirow{4}{*}{$\begin{array}{c}\text { Emergence }(\mathrm{E}) \\
\text { Tillering }(\mathrm{T}) \\
50 \% \text { at } \mathrm{E}+50 \% \text { at } \mathrm{T}\end{array}$} & 34 & 34 & 34 \\
\hline & & 34 & 34 & 34 \\
\hline & & 34 & 34 & 34 \\
\hline & & & & \\
\hline
\end{tabular}




\section{International Journal of Environmental Sciences \& Natural Resources}

\begin{tabular}{|c|l|l|l|}
\hline & Mean & 34 & 34 \\
\hline Control & 34 & & \\
\hline Sorghum water extract & 34 & & \\
\hline Hand weeding & 34 & & \\
\hline Sorghum water extract & 34 & & \\
\hline Herbicide application & 34 & & \\
\hline Sorghum water extract & 34 & & \\
\hline
\end{tabular}

LSD value for concentration at $P \leq 0.05=N S$.

LSD value for Application time at $\mathrm{P} \leq 0.05=\mathrm{NS}$.

Mean sharing the same letters are non significant at $P \leq 0.05$ using LSD test.

Statistical analysis of the data showed that sorghum extract type (SET), Concentration(C), application time (AT), control vs. treated, hand weeding ( $\mathrm{HW}$ ) vs. treated and herbicide application (HA) vs. treated significantly affected thousand grain weight, while all possible interactions were nonsignificant (Table 5). Mean values for sorghum water extract (SWE) concentration showed that higher concentration (1:3) resulted in more thousand grain weight (47.17 g). Decreasing concentration decreased thousand grain weights and minimum thousand grain weight $(40.61 \mathrm{~g})$ was recorded at 1:5. HW plot produced significantly maximum thousand grain weight (50 g) as compared with SWE (44 g). Whereas the plot treated

Table 6: Thousand grain weight of wheat as affected by sorghum water extract type, concentration and application time.

\begin{tabular}{|c|c|c|c|c|}
\hline \multirow{2}{*}{$\begin{array}{c}\text { Concentration } \\
\text { Herbage(kg):Water(L) }\end{array}$} & \multirow{2}{*}{ Application Time } & \multicolumn{2}{|c|}{ Extract Type } & \multirow{2}{*}{ Mean } \\
\hline & & Leaf water extract & Stem water extract & \\
\hline $1: 3$ & Emergence (E) & 49 & 47 & 48 \\
\hline $1: 3$ & Tillering $(\mathrm{T})$ & 48 & 46 & 47 \\
\hline $1: 3$ & $50 \%$ at $\mathrm{E}+50 \%$ at $\mathrm{T}$ & 47 & 45 & 46 \\
\hline $1: 4$ & Emergence (E) & 45 & 43 & 44 \\
\hline $1: 4$ & Tillering $(\mathrm{T})$ & 45 & 43 & 44 \\
\hline $1: 4$ & $50 \%$ at $\mathrm{E}+50 \%$ at $\mathrm{T}$ & 42 & 41 & 42 \\
\hline $1: 5$ & Emergence (E) & 43 & 41 & 42 \\
\hline $1: 5$ & Tillering $(\mathrm{T})$ & 42 & 40 & 41 \\
\hline $1: 5$ & $50 \%$ at $\mathrm{E}+50 \%$ at $\mathrm{T}$ & 40 & 38 & 39 \\
\hline $1: 3$ & & 48 & 46 & 47 \\
\hline $1: 4$ & & 44 & 42 & 43 \\
\hline \multirow[t]{4}{*}{$1: 5$} & & 42 & 39 & 41 \\
\hline & \multirow{3}{*}{$\begin{array}{c}\text { Emergence }(\mathrm{E}) \\
\text { Tillering }(\mathrm{T}) \\
50 \% \text { at } \mathrm{E}+50 \% \text { at } \mathrm{T}\end{array}$} & 46 & 44 & 45 \\
\hline & & 45 & 43 & 44 \\
\hline & & 43 & 41 & 42 \\
\hline \multicolumn{2}{|c|}{ Mean } & 45 & 43 & \\
\hline
\end{tabular}




\section{International Journal of Environmental Sciences \& Natural Resources}

\begin{tabular}{|c|c|c|c|}
\hline Control & 37 & & \\
\cline { 2 - 4 } Sorghum water extract & 44 & & \\
\hline Hand weeding & 50 & & \\
\hline Sorghum water extract & 44 & & \\
\hline Herbicide application & 51 & & \\
\hline Sorghum water extract & 44 & & \\
\hline
\end{tabular}

LSD value for concentration at $P \leq 0.05=2.115685$.

LSD value for Application time at $P \leq 0.05=2.115685$.

Mean sharing the same letters are non significant at $P \leq 0.05$ using LSD Test.

\section{Grain yield (kg ha-1)}

Statistical analysis of the data showed that sorghum extract type (SET), Concentration(C) application time (AT), control vs. treated, hand weeding (HW) vs. treated and herbicide application (HA) vs. treated significantly affected grain yield, while all possible interactions were found non-significant (Table 5).Mean values for sorghum water extract (SWE) concentration showed that higher concentration (1:3) resulted in more grain yield (3575 $\mathrm{kg} \mathrm{ha}^{-1}$ ). Decreasing concentration decreased grain yield and minimum grain yield (3134 $\left.\mathrm{kg} \mathrm{ha}^{-1}\right)$ was recorded at 1:5. HW plot produced significantly more grain yield (3900 kg ha-1) as compared with SWE (3354 $\left.\mathrm{kg} \mathrm{ha}^{-1}\right)$.Whereas the plot treated with SWE produced significantly more grain yield (3354 $\left.\mathrm{kg} \mathrm{ha}^{-1}\right)$ as compared with control plot $\left(2224 \mathrm{~kg} \mathrm{ha}^{-1}\right.$. In case of SET the mean values showed that maximum grain yield (3379 $\mathrm{kg} \mathrm{ha}^{-1}$ ) was produced by the plot treated with leaf water extract (LWE), whereas the minimum grain yield (3330 $\left.\mathrm{kg} \mathrm{ha}^{-1}\right)$ was produced by plot treated with stem water extract (SWE). Mean values for AT showed that SWE applied at emergence produced maximum grain yield (3404 $\left.\mathrm{kg} \mathrm{ha}^{-1}\right)$, while minimum grain yield (3280 kg ha $^{-1}$ ) was recorded when SWE applied half at emergence and half at tillering stage.

\section{Biological yield ( $\left.\mathrm{kg} \mathrm{ha}^{-1}\right)$}

Statistical analysis of the data revealed that sorghum extract type (SET), Concentration(C), application time (AT), control vs. treated, hand weeding (HW) vs. treated and herbicide application (HA) vs. treated significantly affected biological yield, while Table 7: Grain yield of wheat as affected by sorghum water extract type, concentration and application time.

\begin{tabular}{|c|c|c|c|c|}
\hline \multirow{2}{*}{$\begin{array}{c}\text { Concentration } \\
\text { Herbage(kg):Water(L) }\end{array}$} & \multirow{2}{*}{ Application Time } & \multicolumn{2}{|c|}{ Extract Type } & \multirow{2}{*}{ Mean } \\
\cline { 3 - 5 } & & Leaf water extract & Stem water extract & 3628 \\
\hline $1: 3$ & Emergence (E) & 3660 & 3597 & 3604 \\
\hline $1: 3$ & Tillering (T) & 3627 & 3581 & 3494 \\
\hline $1: 3$ & $50 \%$ at E +50\% at T & 3516 & 3471 & 3393 \\
\hline $1: 4$ & Emergence (E) & 3411 & 3356 & 3381 \\
\hline $1: 4$ & Tillering (T) & 3406 & 3264 & 3283 \\
\hline $1: 4$ & $50 \%$ at E +50\% at T & 3301 & 3175 & 3193 \\
\hline $1: 5$ & Emergence (E) & 3211 & 3107 & 3147 \\
\hline
\end{tabular}


International Journal of Environmental Sciences \& Natural Resources

\begin{tabular}{|c|c|c|c|c|}
\hline $1: 5$ & $50 \%$ at $\mathrm{E}+50 \%$ at $\mathrm{T}$ & 3088 & 3040 & 3064 \\
\hline $1: 3$ & & 3601 & 3550 & 3575 \\
\hline $1: 4$ & & 3373 & 3332 & 3352 \\
\hline $1: 5$ & & 3162 & 3107 & 3135 \\
\hline & Emergence (E) & 3427 & 3382 & 3405 \\
\hline & Tillering (T) & 3407 & 3348 & 3377 \\
\hline & $50 \%$ at $\mathrm{E}+50 \%$ at $\mathrm{T}$ & 3302 & 3259 & 3280 \\
\hline \multicolumn{2}{|c|}{ Mean } & 3379 & 3330 & \\
\hline \multicolumn{2}{|c|}{ Control } & 2224 & & \\
\hline \multicolumn{2}{|c|}{ Sorghum water extract } & 3354 & & \\
\hline \multicolumn{2}{|c|}{ Hand weeding } & 3900 & & \\
\hline \multicolumn{2}{|c|}{ Sorghum water extract } & 3354 & & \\
\hline \multicolumn{2}{|c|}{ Herbicide application } & 3976 & & \\
\hline \multicolumn{2}{|c|}{ Sorghum water extract } & 3354 & & \\
\hline
\end{tabular}

LSD value for concentration at $P \leq 0.05=58.89661$

LSD value for Application time at $P \leq 0.05=58.89661$

Mean sharing the same letters are non significant at $P \leq 0.05$ using LSD test

Table 8: Biological yield of wheat as affected by sorghum water extract type, concentration and application time.

\begin{tabular}{|c|c|c|c|c|}
\hline \multirow{2}{*}{$\begin{array}{c}\text { Concentration } \\
\text { Herbage(kg):Water(L) }\end{array}$} & \multirow{2}{*}{ Application Time } & \multicolumn{2}{|c|}{ Extract Type } & \multirow{2}{*}{ Mean } \\
\hline & & Leaf water extract & Stem water extract & \\
\hline $1: 3$ & Emergence (E) & 9857 & 9664 & 9760 \\
\hline $1: 3$ & Tillering (T) & 9790 & 9577 & 9683 \\
\hline $1: 3$ & $50 \%$ at $\mathrm{E}+50 \%$ at $\mathrm{T}$ & 9574 & 9371 & 9473 \\
\hline $1: 4$ & Emergence (E) & 9541 & 9270 & 9406 \\
\hline $1: 4$ & Tillering (T) & 9488 & 9159 & 9324 \\
\hline $1: 4$ & $50 \%$ at $\mathrm{E}+50 \%$ at $\mathrm{T}$ & 9272 & 8840 & 9056 \\
\hline $1: 5$ & Emergence (E) & 9141 & 8735 & 8938 \\
\hline $1: 5$ & Tillering (T) & 9049 & 8411 & 8730 \\
\hline $1: 5$ & $50 \%$ at $\mathrm{E}+50 \%$ at $\mathrm{T}$ & 8879 & 7747 & 8313 \\
\hline $1: 3$ & & 9740 & 9537 & 9639 \\
\hline $1: 4$ & & 9434 & 9090 & 9262 \\
\hline \multirow[t]{4}{*}{$1: 5$} & & 9023 & 8298 & 8660 \\
\hline & \multirow{3}{*}{$\begin{array}{c}\text { Emergence }(\mathrm{E}) \\
\text { Tillering }(\mathrm{T}) \\
50 \% \text { at } \mathrm{E}+50 \% \text { at } \mathrm{T}\end{array}$} & 9513 & 9223 & 9368 \\
\hline & & 9442 & 9049 & 9246 \\
\hline & & 9242 & 8653 & 8947 \\
\hline \multicolumn{2}{|c|}{ Mean } & 9399 & 8975 & \\
\hline \multicolumn{2}{|c|}{ Control } & 7781 & & \\
\hline \multicolumn{2}{|c|}{ Sorghum water extract } & 9187 & & \\
\hline \multirow{2}{*}{\multicolumn{2}{|c|}{$\begin{array}{c}\text { Hand weeding } \\
\text { Sorghum water extract }\end{array}$}} & 10317 & & \\
\hline & & 9187 & & \\
\hline \multicolumn{2}{|c|}{ Herbicide application } & 10558 & & \\
\hline \multicolumn{2}{|c|}{ Sorghum water extract } & 9187 & & \\
\hline
\end{tabular}

LSD value for concentration at $P \leq 0.05=343.4659$.

LSD value for Application time at $\mathrm{P} \leq 0.05=343.4659$.

Mean sharing the same letters are non significant at $P \leq 0.05$ using LSD test. 


\section{International Journal of Environmental Sciences \& Natural Resources}

Table 9: Harvest index of wheat affected by sorghum extract type, concentration and application time.

\begin{tabular}{|c|c|c|c|c|}
\hline \multirow{2}{*}{$\begin{array}{c}\text { Concentration } \\
\text { Herbage }(\mathrm{kg}): \text { Water(L) }\end{array}$} & \multirow{2}{*}{ Application Time } & \multicolumn{2}{|c|}{ Extract Type } & \multirow{2}{*}{ Mean } \\
\hline & & Leaf water extract & Stem water extract & \\
\hline $1: 3$ & Emergence (E) & 37 & 37 & 37 \\
\hline $1: 3$ & Tillering (T) & 37 & 37 & 37 \\
\hline $1: 3$ & $50 \%$ at $\mathrm{E}+50 \%$ at $\mathrm{T}$ & 37 & 37 & 37 \\
\hline $1: 4$ & Emergence (E) & 36 & 37 & 36 \\
\hline $1: 4$ & Tillering (T) & 36 & 37 & 36 \\
\hline $1: 4$ & $50 \%$ at $\mathrm{E}+50 \%$ at $\mathrm{T}$ & 36 & 37 & 36 \\
\hline $1: 5$ & Emergence (E) & 35 & 36 & 36 \\
\hline $1: 5$ & Tillering (T) & 35 & 37 & 36 \\
\hline $1: 5$ & $50 \%$ at $\mathrm{E}+50 \%$ at $\mathrm{T}$ & 35 & 39 & 37 \\
\hline $1: 3$ & & 37 & 37 & 37 \\
\hline $1: 4$ & & 36 & 37 & 36 \\
\hline \multirow[t]{4}{*}{$1: 5$} & & 35 & 38 & 36 \\
\hline & \multirow{3}{*}{$\begin{array}{c}\text { Emergence }(\mathrm{E}) \\
\text { Tillering }(\mathrm{T}) \\
50 \% \text { at } \mathrm{E}+50 \% \text { at } \mathrm{T}\end{array}$} & 36 & 37 & 36 \\
\hline & & 36 & 37 & 37 \\
\hline & & 36 & 38 & 37 \\
\hline \multicolumn{2}{|c|}{ Mean } & 36 & 37 & \\
\hline \multicolumn{2}{|c|}{ Control } & 29 & & \\
\hline \multicolumn{2}{|c|}{ Sorghum water extract } & 37 & & \\
\hline \multirow{2}{*}{\multicolumn{2}{|c|}{$\begin{array}{c}\text { Hand weeding } \\
\text { Sorghum water extract }\end{array}$}} & 38 & & \\
\hline & & 37 & & \\
\hline \multicolumn{2}{|c|}{ Herbicide application } & 38 & & \\
\hline \multicolumn{2}{|c|}{ Sorghum water extract } & 37 & & \\
\hline
\end{tabular}

LSD value for concentration at $P \leq 0.05=N S$.

LSD value for Application time at $\mathrm{P} \leq 0.05=\mathrm{NS}$.

Mean sharing the same letters are non significant at $P \leq 0.05$ using LSD test.

\section{Discussion}

Data recorded on number of weeds at 100 days after sowing revealed that all weed control treatments significantly affected the weeds density. Minimum numbers of weeds were noted in herbicides sprayed plots, while maximum number of weeds were recorded in plots treated with sorghum water extract at lower concentration applied half at emergence and half at tillering stage. It was noted that as the concentration of sorghum water extract increased, the number of weeds decreased accordingly. The results are in agreement with the finding of Bhowmik and Dolt (1982) and Wajid et al. [13] they reported that sorghum water extract suppressed weeds germination and growth more than that of wheat.

Lower productive and higher non productive tillers were recorded in control and in plots where less concentration of sorghum water extract was applied half at emergence and half at tillering stage. The lower number of productive tillers and higher number of non productive tillers may be due to dense population and greater competition with weeds for light, nutrients and space. The herbicide application and sorghum water extract at higher concentration (1:3) applied at emergence produced less number of non productive tillers. Less number may be due to less competition between plants for up taking and utilizing nutrients efficiently at tiller formation stage and later stage.

Thousand grain weight is highly correlated with photosynthetic rate or source of assimilates during seed fill duration. It is one of the most important yield components and contributes significantly to the final grain yield. Higher grain weight was recorded in plots treated with herbicides and sorghum water extract at higher concentration (1:3) applied at emergence as compared with control. The probable reason for higher yield in weed control plots might be that herbicides and sorghum water extract reduced the wheat plant competition with weeds for nutrients, light, moisture and space thus maximized the utilization of nutrients from the soil which finally developed heavy seeds. Our findings agree with Anwar S [14] who reported heavier grains with higher application of sorghum water extract.

Grain yield of a crop is final goal towards which all the efforts 
are directed. Data recorded on grain yield indicate that all that all weed control treatments significantly increased grain yield. Both herbicide application and sorghum water extract at higher concentration applied at emergence produced the higher grain yield, while minimum grain yield was recorded in control. These results are in agreement with Cheema et al. [15] who showed that increasing the sorghum water extract from $50 \%$ to $100 \%$ improved grain yield by $14 \%$. This may be due to the fact that all yield components were higher in plants treated with herbicides and sorghum water extract at higher concentration applied at emergence which increased grain $\mathrm{ha}^{-1}$. The possible reason for high grain yield in different wed control treatments may be that increase availability of nutrients and reduced competition stimulated vegetative growth which resulted in better spike population and more grains per spike and ultimately higher grain yield.

Biological, yield which provides an important physiological basis for grain yield of a crop was significantly affected by weed control treatments. Maximum biological yield was recorded in plots treated with herbicide application followed by plots treated with sorghum water extract at higher concentration (1:3) applied at emergence, while minimum yield was recorded in plots treated with less sorghum water extract concentration (1:5). The increase in yield is attributed to more tillers per unit area and luxurious vegetative growth. More biological yield in case of herbicide application and in plots with sorghum water extract applied at lower concentration may be due to the fact that decrease in weeds population due to herbicide application and sorghum water extract applied at higher concentration resulted in stimulation of vegetative growth which increased cell size, plant height, spike population and thus resulting in overall increase in biological yield. Similar results were obtained by Mukhopandyay and Gosh (1980) who reported that all chemical treatments were superior in yield as compared with control.

\section{Summary}

To study the effect of sorghum water extract type, concentration, and application time on weed density and yield of wheat an experiment was conducted at Agriculture Research Farm, The University of Agriculture Peshawar, Pakistan during winter season 2014-15. The experiment was laid out in a randomized complete block design (RCBD) having three replication. A plot size of $3 \mathrm{~m} \times 1.8 \mathrm{~m}$ (L x W) was used. Each plot was consist of six rows having $30 \mathrm{~cm}$ row to row distance. Seed was sown at the rate of $120 \mathrm{~kg}$ ha-1. Different concentration (1:3) i.e. (1 kg plant herbage and 3 liters of water), (1:4) i.e. (1 kg plant herbage and 4 liters of water) and 1:5 (1 kg plant herbage and 5 liters of water) of sorghum stem and leaf extract was sprayed at three stages full at Feekes 1 (Emergence), full at Feekes 2 (Tillering), and half at Feekes 1 (Emergence) plus half at Feekes 2 (Tillering). The experiment was also comprised of control (no weed control), hand weeding and herbicides application. All other agronomic practices were applied uniformly. Data was recorded on Weed density, Productive tillers ,Non-productive tillers, Days to physiological maturity, Grain filling duration, Thousand grain weight (g),Grain yield, Biological yield and Harvest index.

Number of weeds recorded at 100 days after sowing were significantly affected by weed control treatments. Minimum number of weeds (24) were recorded in hand weeding plot followed by herbicides (29) treated plots, while maximum (183) weeds were recorded in control. Sorghum water extracts treated plots also recorded less (134) number of weeds as compared with control (183). Number of productive tillers m-2 were significantly affected by weed control treatments. Maximum (379) number of productive tillers $\mathrm{m}-2$ was observed in herbicides sprayed plots, while minimum (282) number of productive tillers was recorded in control. Non productive tillers were also significantly affected by weed control treatments. Maximum number of non productive tillers was recorded in control (34) and plots treated with less concentration of sorghum water extract applied half at emergence and half at tillering stage (31), while minimum number of non productive tillers was observed in herbicides sprayed plots (21).

All the weed control treatments had no significant effect on grain filling duration and days to physiological maturity same (34) days to grain fill duration and physiological maturity (155) days were recorded in different weed control plots. Same number of days (34) for grain filling duration and (155) days to physiological maturity were recorded in control. Thousand grain weight and biological yield were significantly affected by weed control treatments. Maximum grain weight (51 g) and (10558 $\mathrm{kg} \mathrm{ha}^{-1}$ ) biological yield was observed in plots treated with herbicides. Similarly sorghum water extract application at higher concentration also increased ( $44 \mathrm{~g}$ ) grain weight and (9187 $\mathrm{kg} \mathrm{ha}^{-1}$ ) biological yield as compared with (37 g) grain weight and (7781 $\mathrm{kg} \mathrm{ha}^{-1}$ ) biological yield in control. While minimum ( $37 \mathrm{~g}$ ) grain weight and $\left(7781 \mathrm{~kg}^{-1}\right)$ biological yield was recorded in control. All weed control treatments had significant effect on grain yield, herbicide treated plots gave maximum (3976 $\mathrm{kg}^{-1}$ ) grain yield. Similarly sorghum water extract at higher concentration also increased (3354 kg ha-1) grain yield as compared with control (2224 $\left.\mathrm{kg} \mathrm{ha}^{-1}\right)$.

\section{Conclusion}

It is concluded that among all weed control treatments, herbicides gave best results but the allelopathic effects of sorghum extracts were also prominent and followed the herbicides. In case of sorghum concentration, higher concentration (1:3) applied at emergence gave best result. Sorghum extract affect weeds and wheat growth due to either inhibitory or stimulatory effects depending upon the quantity of sorghum and stage of growth of wheat. So the allelopathic potential of sorghum and its effects on weed flora should be further explored as less expensive, safe and harmless method of weeds controlling wheat. 


\section{Recommendations}

It is recommended that sorghum leaf water extract at higher concentration 1:3 should be applied at emergence for controlling of weeds and improving yield of wheat.

\section{References}

1. Blackshaw RE, JT Odonovan, KN Harker, GW Clayton, RN Stougaard (2006) Reduced herbicide doses in field crops: A review. Weed Biol \& Manag 6(1): 10-17.

2. Asalam V, W Kebapci, S Gucel, E Cetin, E Altundag (2014) Land degradation in the lower Euphrates sub region of Turkey. J Environ Biol 75: 371-363.

3. MINFA (2012) Government of Pakistan. Ministry of National Food Security and Research, Agricultural statistics of Pakistan.

4. Ozturk M, U Kebapci, S Gucel, E Cetin, E Altundag (2012) Biodiversity and land degradation in the lower Euphrates sub region of Turkey. J Environ Biol 33(2): 311-323.

5. Marwat KB, M Saeed, Z Hussain, B Gul, H Rashid (2008) Study of various herbicides for weed control in wheat under irrigated conditions. Pak J Weed Sci Res 14(1/2): 1-8.

6. Kordali S, A Cakir, TA Akcin, E Mete, A Akcin et al. (2009) Antifungal and herbicidal properties of essential oils and $n$-hexane extracts of Achillea gypsicola Hub Mor. and Achilleabiebersteinii afan. (Asteraceae). Ind Crop Prod 29: 562-570.

7. Bhowmik PC, J Inderjit (2003) Challenges and opportunities in implementing allelopathy for natural weed management. Crop Prot 22 (4): 661-671.
8. Jabran K, ZA Cheema, M Farooq, M Hussain (2010) Lower doses of pendimethalin mixed with allelopathic crop water extracts for weed management in canola (Brassica napus). Int J Agric Biol 12: 335-340.

9. Farooq M, K Jabran, H Rehman, M Hussain (2008) Allelopathic effects of rice on seedling development in wheat, oat, barley and berseem. Allelopathy J 22 (2): 385-390.

10. MT Jan, P Shah, PA Hollington, MJ Khan, Q Sohail (2009) Agriculture Research: Design and Analysis, A Monograph. NWFP Agric Univ Peshwar, Pakistan.

11. Ahmad Khan, Mohd Jan, Mohd Arif, B Marwat Jan Amanullah (2008) Crop stand of wheat as affected by nitrogen sources and tillage systems. Pak J Bot 45(6): 1103-1222.

12. Ahmad Khan, Mohammad Tariq Jan, Muhammad Arif, Khan Bahadar Marwat, Amanullah Jan (2008) Phenology and crop stand of wheat as affected by nitrogen sources and tillage systems. Pak J Bot 40(3): 11031112.

13. Shazma Anwar, Wajid Ali Shah, Jehan Bakht, Nadia Jabeen (1985) Comparison of sorghum extracts chemical and hand weeding management in wheat crop J Agron 3 (1): 59-67.

14. Anwar S, Shah WA, Shafi M, Bakht (2003) Efficiency of sorgaab (sorghum water extract) and herbicides for weed control in wheat crop. Pak J Weed Sci Res 9(3\&4):161-170.

15. Cheema ZA, A Khaliq, M Mubeen (2003) Response of wheat and winter weeds to foliar application of different plant water extracts of sorghum (S. bicolor). Pak J Weed Sci Res 9(1-2): 89-97.

\section{Your next submission with Juniper Publishers will reach you the below assets}

- Quality Editorial service

- Swift Peer Review

- Reprints availability

- E-prints Service

- Manuscript Podcast for convenient understanding

- Global attainment for your research

- Manuscript accessibility in different formats

( Pdf, E-pub, Full Text, Audio)

- Unceasing customer service

Track the below URL for one-step submission https://juniperpublishers.com/online-submission.php 